\title{
Autoestima en pacientes drogodependientes
}

\section{Self-esteem in drug-dependent patients}

\section{Autoestima en pacientes}

\section{Cindy Gabriela Vásquez Vera ${ }^{1}$}

\section{Jisson Oswaldo Vega Intriago ${ }^{2}$}

${ }^{1}$ Instituto de Posgrado de la Universidad Técnica de Manabi. Ecuador cvasquez1232@utm.edu.ec. Código Orcid https://orcid.org/0000-0003-4871-1605

${ }^{2}$ Instituto de Posgrado de la Universidad Técnica de Manabi. Ecuador. jisson.vega@utm.edu.ec. Código Orcid https://orcid.org/0000-0001-5727-8837

Contacto: cvasquez1232@utm.edu.ec

\section{Resumen}

El manejo de la autoestima ayuda a prevenir conductas de riesgo y permite una adaptación del individuo al medio en el cual se desenvuelve, en el presente trabajo, se propuso como objetivo general describir el nivel de autoestima de los pacientes de los Centros especializados en tratamiento de alcohol y drogas "Nuevo Surco" y “CENTRADRO". La investigación realizada fue de tipo descriptivo, para la recolección de información pertinente se aplicó la escala de autoestima Coopersmith para adultos. Donde los resultados en su escala general alcanzaron en su mayoría un nivel medio alto, en ambas instituciones

Palabras clave: Autovalía, consumo, escala de autoestima

\section{Sumary}

Self-esteem management helps prevent risky behaviors and allows an individual to adapt to the environment in which they operate, the objective of this study is to describe the level of self-esteem of patients at the Centers specialized in alcohol and drug treatment "Nuevo Surco" and "CENTRADRO". The research was carried out descriptively, for the collection of pertinent information the Coopersmith self-esteem scale for adults was applied. Where the results on their general scale mostly reached a medium-high level, in both institutions.

Keyword: Self-worth, consumption, self-esteemscale.

\section{Introducción}


La autoestima desempeña un papel fundamental en el desarrollo del individuo y sus áreas de interacción, ya que refleja el valor que cada individuo posee, mostrando la confianza sobre sí y reconociendo su autovalía. En pacientes drogodependientes estos niveles de autoestima van a influir en sus emociones y por consiguiente en sus comportamientos, repercutiendo sobre sí mismo y en sus áreas de desenvolvimiento, como el social y familiar.

En el presente estudio se evidencia el nivel de la autoestima de los pacientes drogodependientes de dos Centros especializados en el tratamiento de alcohol y drogas (Cetad), los resultados obtenidos en la aplicación de la escala de autoestima Coopersmith para adultos quedan registrados dentro del articulo para contribuir a las futuras investigaciones sobre la misma temática ya que no existen suficientes investigaciones sobre el presente tema investigado.

El consumo de sustancias un factor que afecta considerablemente a la población, siendo el consumo de alcohol un problema que va en aumento en el área de la salud, principalmente en adolescentes, donde se lo relaciona con la autoestima como factor protector ante el consumo, debido a que afecta a la adquisición o modificación de hábitos saludables (Telumbre et al., 2018).

Dentro de las investigaciones sobre consumo de sustancias prevalecen las realizadas al consumo de alcohol enfocadas en la autoestima, a esto,(Gámez \& Guzmán, 2017) hicieron una investigación en Monterrey, México, donde realizaron un estudio con 668 estudiantes para relacionar el nivel de autoestima con el consumo de alcohol, por ello refieren los siguiente resultados: "Con respecto a la autoestima, en la mayor parte de los adolescentes tiene alta autoestima (76.2\%), seguida de la autoestima promedio (17.4\%) y de la autoestima baja (6.4\%)". Basándose en el modelo de regresión logística para la autoestima y la prevalencia del consumo de alcohol en adolescente descubrieron en mencionado estudio que si existe una relación importante.

El nivel bajo de la autoestima es un factor que marca prevalencia en consumidores de alcohol, a esto (Cuero, 2017) dentro de su investigación realizada en Quito concluye: “el nivel de autoestima que prevalece entre los estudiantes, que se inician en el consumo de alcohol es la baja autoestima. Tanto en los alumnos hombres como en las mujeres"

Por otro lado, en un estudio realizado en México a 1,245 adolescentes entre 12 y 17 años, se analizó y se concluyó aportaciones muy importantes entre ellas que la influencia familiar, el apoyo familiar, en funcionamiento familiar y el apoyo social influye en la autoestima del adolescente y en su consumo de alcohol, 
ya que al ser seres biopsicosociales aprenden de su entorno y se relacionan e influyen de el mismo (Villarreal \& Sanchez, 2010).

Así también, es importante mencionar que hay eventos que pueden generen traumas desde la infancia, por la carga que aportan podían generar emociones negativas que pueden llegar a disminuir la autoestima, y el evento traumático configurarse (Matrangolo et al., 2017).

Independientemente de cuales sean los factores socioeconómicos el consumo ataca a la población ecuatoriana, en estudios realizados basados en investigaciones previas se detectaron que varios son aquellos que inciden en este comportamiento de abuso de sustancia como lo son carencia de apoyo familiar, influencias sociales y desconocimiento de verdadero efecto negativo que causan las mismas (Loor \& Hidalgo, 2018).

Siguiendo con lo anteriormente mencionado es significativo conocer los factores de protección de los pacientes, ya que será un factor clave para la estabilidad emocional de las personas que consumen sustancias psicoactivas, debido a que al reconocerlas podrán controlar sus impulsos adictivos. La autoestima siendo el factor protector que contribuye a que el paciente se cuide en las esferas: biológica, psicológica y social (Liberini et al., 2016).

Como se ha mencionado antes la relevancia de conocer los factores de protección, se obtuvieron diferencias estadísticas, que las creencias irracionales entre ellas la indefensión, podría ser uno de los motivos que lleven a los adolescentes a probar sustancias psicoactivas, ya que no poseen recursos para manejar problemas de la vida diaria o sucumbir a la presión del grupo y consumir (Carbonero et al., 2010).

En la adolescencia el consumo de sustancias se convierte en un problema, que afecta a todas las esferas en las que se desarrolla. Por eso el profesional encargado deberá someter a una evaluación exhaustiva a cada paciente para conocer en la etapa del consumo se encuentra y la intervención o modelo terapéutico a seguir (Tena et al., 2018)

Existen varios modelos para estudiar a las adicciones entre ellos (Apud \& Romani, 2016) menciona:

El modelo biomédico, con sus características y los problemas que enfrenta al constreñir la adicción bajo un modelo neurobiológico. En segundo lugar, el modelo biopsicosocial, donde se integran variables psicológicas y de contexto. Este modelo provoca un reacomodamiento del modelo biomédico, pero también genera perspectivas críticas a su enfoque neurobiológico. En tercer lugar, el modelo sociocultural, donde, desde la investigación cualitativa en ciencias sociales, se focaliza en la importancia de la cultura y el contexto social. Pág. 123 
La terapia cognitivo conductual en el proceso de pacientes drogodependientes es de suma importancia ya que se enfoca en restaurar factores adaptativos, de autoconciencia y autodeterminación de los mismos. (Moreno, 2016)

El consumo de sustancias es un factor que no solo afecta a la persona que lo consume sino también a su familia y hasta su círculo social, es decir, que la intervención a darse con dichos pacientes debe ser bien planificada y la selección de los métodos y técnicas a emplearse fundamentados y validados para así aumentar su confiabilidad. (Moreno, 2016)

Por otro lado, tomando en cuenta la autoestima como factor importante en pacientes drogodependientes, ya que refleja el valor que tiene cada uno sobre su forma de ser, haciendo hincapié a esto en la opinión de Santrock citado en (Naranjo,2007) Quien expresa que "La autoestima también se refiere a la autovalía o a la autoimagen, y refleja la confianza global del individuo y la satisfacción de sí mismo” (p. 3).

Una de las principales características del abuso de consumir drogas es el afán por conseguir las mismas sin importar las consecuencias que estas traigan consigo, específicamente si las mismas se tornan negativas, es por esto que se requiere de un tratamiento multidisciplinario (Loor \& Hidalgo, 2018).

En un estudio realizado en México con el tema "El rol de la autoestima en el consumo moderado de drogas en la adolescencia" demuestra la controversia en cuanto a si existe relación entre la autoestima y el consumo de drogas dentro de la discusión final de dicha investigación menciona:

"la relación de la autoestima y el consumo de sustancias se debe analizar desde una perspectiva multidimensional... la autoestima familiar y la autoestima académica tienden a inhibir las conductas que implican consumo de sustancias (alcohol y drogas) mientras que la autoestima social está asociada con un mayor consumo de sustancias.” (Misitu Ochoa, 2003, p. 296).

Es decir, no solo la autoestima general sino la autoestima dividida dependiendo su interacción ya sea por el lado de la familia, responsabilidades y área social, donde cada uno representaría o no relación al consumo.

Mientras que en un estudio realizado en Perú con tema autoestima y autoeficacia en pacientes adictos en el centro de rehabilitación de ÑANA. Dentro de sus resultados Castro Deza (2012) menciona "la autoestima se incrementa conforme el paciente va avanzando en su programa de tratamiento" (p.20) es decir a medida que el paciente va cumpliendo con los procesos requeridos dentro de los programas establecidos en la institución el autoestima va creciendo fortaleciendo la valía que tiene sobre sí mismo. 
Por otro lado, en Ecuador sobre la prevención del consumo de drogas, en Machala se realizó una investigación de tinte bibliográfico científico enfocada en la prevención con el tema: Una propuesta de prevención del consumo de drogas en adolescentes, donde dentro de la misma mencionan: "la autoestima como un factor de riesgo que se debe tomar en cuenta para la prevención del consumo de drogas” (2018, p. 8).

Para determinar el nivel de autoestima se han validado diversas escalas dentro de las cuales se puede mencionar la escala de autoestima de Coopersmith que inicialmente fue creado en versión Escolar por Stanley Coopersmith, el inventario de autoestima de Coopersmith versión adulta consta de 25 ítems donde genera un puntaje total y separado en el área de sí mismo que hace referencia a actitudes de su autopercepción, en el área social frente a compañeros, amigos y en el área familiar con relación a su convivencia familiar.

Se ha hablado de como la influencia social y familiar influyen en la autoestima de los individuos con consumo problemático de drogas, a esto el presente estudio tuvo como objetivo general describir el nivel de autoestima de los pacientes de los CETAD "Nuevo surco" y "CENTRADRO” donde se identifica el nivel de la dimensión de sí mismo, dimensión social y dimensión familiar de la autoestima en los pacientes.

\section{Materiales y métodos}

El presente estudio es cuantitativo de tipo descriptivo mediante la aplicación de la escala de autoestima de Coopersmith, los mismos que fueron ingresados al programa Excel donde se obtuvieron los resultados de la escala y el procesamiento de los resultados se lo realizó mediante el programa SPSS, la población fue de 34 pacientes internos en centros especializados en tratamiento de alcohol y drogas, donde se trabajó con 21 pacientes internos del CETAD “CENTRADRO” y 13 del CETAD "Nuevo surco", se incluyeron a los pacientes mayores de 18 años, hombres, con consumo problemático de alcohol y drogas, se excluyeron a los pacientes con patología dual y con un proceso terapéutico mayor a 3 meses, los participantes firmaron los respectivos consentimientos informados. Declaración de no tener conflicto de interés y confidencialidad de la información.

\section{Resultados}

Presentación y análisis que se obtuvo de los datos de la escala de autoestima aplicada a los internos de Los Centro Especializado en Tratamiento de alcohol y drogas "Nuevo surco" y “CENTRADRO” 2021 
Tabla 1. Porcentaje de, moda de la escala de autoestima aplicada a los internos del Centro Especializado en Tratamiento de alcohol y drogas "Nuevo surco" 2021

\section{Estadísticos}

\begin{tabular}{llc|c|c|c} 
& & Si Mismo & Social & Familia & Escala General \\
\hline $\mathrm{N} \quad$ Válido & 13 & 13 & 13 & 13 \\
\cline { 2 - 6 } & Perdidos & 0 & 0 & 0 & 0 \\
\hline \multicolumn{2}{l|}{ Moda } & 4,00 & 3,00 & 3,00 & 3,00 \\
\hline
\end{tabular}

De acuerdo a los resultados se determinó que la moda dentro de la escala general es de 3 equivalente a un nivel de autoestima medio alto, variando en la moda de sí mismo que en su mayoría fue 4 lo que representa un nivel de autoestima alto, misma que va a servir de base para fortalecer otras áreas del paciente que presentan déficit.

Tabla 2. Porcentaje de moda de la escala de autoestima aplicada a los internos del Centro Especializado en Tratamiento de alcohol y drogas "CENTRADRO” 2021

\section{Estadísticos}

\begin{tabular}{lll|l|l|l} 
& & Si Mismo & Social & Familia & Escala General \\
\hline N & Válido & 21 & 21 & 21 & 21 \\
\cline { 2 - 6 } & Perdidos & 0 & 0 & 0 & 0 \\
\hline Moda & 4,00 & 3,00 & 3,00 & 3,00 \\
\hline
\end{tabular}

De acuerdo a los resultados se determinó que la moda dentro de la escala general es de 3 equivalente a un nivel de autoestima medio alto, variando en la moda de si mismo que en su mayoría fue 4 lo que representa un nivel de autoestima alto, misma que va a servir de base para fortalecer otras áreas del paciente que presentan déficit.

Tabla.3 Distribución de frecuencia y porcentaje del nivel de sí mismo de la escala de autoestima aplicada a los de los Centro Especializado en Tratamiento de alcohol y drogas "Nuevo surco" y "CENTRADRO” 2021 


\begin{tabular}{|c|c|c|c|c|c|}
\hline & & Frecuencia & Porcentaje & Frecuencia & Porcentaje \\
\hline \multirow[t]{5}{*}{ Válido } & $\begin{array}{l}\text { Nivel de autoestima } \\
\text { bajo }\end{array}$ & 1 & 7,7 & 0 & 0 \\
\hline & $\begin{array}{l}\text { Nivel de autoestima } \\
\text { medio bajo }\end{array}$ & 2 & 15,4 & 0 & 0 \\
\hline & $\begin{array}{l}\text { Nivel de autoestima } \\
\text { medio alto }\end{array}$ & 3 & 23,1 & 38,1 & 38,1 \\
\hline & $\begin{array}{l}\text { Nivel de autoestima } \\
\text { alto }\end{array}$ & 7 & 53,8 & 13 & 61,9 \\
\hline & Total & 13 & 100,0 & 21 & 100,0 \\
\hline
\end{tabular}

De acuerdo a los resultados del CETAD "Nuevo Surco" se determinó que el mayor número de pacientes poseen un nivel de autoestima alto, con una frecuencia de 7 que representa el 53\% mientras que el porcentaje más bajo con un 7,7\% con una frecuencia de 1 , al ser pacientes internos dentro de un centro al consumo de alcohol y drogas dentro de las cuales se fomenta mediante la terapia vivencial aspectos que resaltan la importancia de sí mismos como consecuencia elevan dichos niveles de autoestima.

Mientras que en el CETAD “CENTRADRO” de acuerdo a los resultados se determinó que el mayor número de pacientes poseen un nivel de autoestima alto, con una frecuencia de 13 que representa el 61,9\% y el nivel de autoestima medio alto representa el porcentaje restante debido a que se anularían tanto el nivel medio bajo como nivel bajo de autoestima ya que ningún paciente posee este nivel en sí mismo, hecho que se fomenta al recibir terapias que incrementan su amor propio.

\begin{tabular}{lll|l|l|l}
\multicolumn{9}{c|}{ Nuevo Surco } & \multicolumn{2}{l}{ CENTRADRO } \\
\hline \multicolumn{2}{c}{ Frecuenci } & Porcentaj \\
& a & e & Frecuencia & Porcentaje \\
\hline Válid & $\begin{array}{l}\text { Nivel de autoestima } \\
\text { o } \\
\text { bajo }\end{array}$ & 7,7 & 0 & 0 \\
\cline { 2 - 6 } & $\begin{array}{l}\text { Nivel de autoestima } \\
\text { medio bajo }\end{array}$ & 7,7 & 2 & 9,5 \\
\hline
\end{tabular}




\begin{tabular}{ll|l|l|l|l}
\hline $\begin{array}{l}\text { Nivel de autoestima } \\
\text { medio alto }\end{array}$ & 61,5 & 11 & 52,4 \\
\hline $\begin{array}{l}\text { Nivel de autoestima } \\
\text { alto }\end{array}$ & 3 & 23,1 & 8 & 31,1 \\
\hline Total & 13 & 100,0 & 21 & 100,0 \\
\hline
\end{tabular}

De acuerdo a los resultados del CETAD "Nuevo Surco" se determinó que el mayor número de pacientes poseen un nivel de autoestima medio alto a nivel social equivalentes al $61,5 \%$ con una frecuencia de 8 , mientras que tanto el nivel de autoestima bajo y medio bajo tienen una frecuencia de 1 , al estar dentro de un centro donde la interacción social va a predominar ya que se reúnen con personas desconocidas a pesar de tener un problema en común mismo que ayuda a integrarse. Mientras que en el CETAD “CENTRADRO” de acuerdo a los resultados se determinó que el mayor número de pacientes poseen un nivel de autoestima medio alto a nivel social equivalentes al $52,4 \% \%$ con una frecuencia de 11 , mientras que tanto el nivel de autoestima medio bajo tienen una frecuencia de 2 equivalente al 9,5\%, al estar dentro de un centro donde la interacción social va a predominar ya que se reúnen con personas desconocidas a pesar de tener un problema en común mismo que ayuda a integrarse, lo que ha eliminado el nivel bajo, ya que ningún paciente posee este nivel dentro del autoestima social.

Tabla 5. Distribución de frecuencia y porcentaje del nivel familiar de la escala de autoestima aplicada a los internos de los Centro Especializado en Tratamiento de alcohol y drogas "Nuevo surco" y "CENTRADRO” 2021

\section{Familia}

\begin{tabular}{|c|c|c|c|c|c|}
\hline & & \multicolumn{2}{|c|}{ Nuevo Surco } & \multicolumn{2}{|c|}{ CENTRADRO } \\
\hline & & $\begin{array}{l}\text { Frecuenci } \\
\text { a }\end{array}$ & $\begin{array}{l}\text { Porcentaj } \\
\text { e }\end{array}$ & Frecuencia & Porcentaje \\
\hline $\begin{array}{l}\text { Válid } \\
\text { o }\end{array}$ & $\begin{array}{l}\text { Nivel de autoestima } \\
\text { bajo }\end{array}$ & 0 & 0 & 2 & 9,5 \\
\hline
\end{tabular}




\begin{tabular}{ll|l|l|l}
\hline $\begin{array}{l}\text { Nivel de autoestima 3 } \\
\text { medio bajo }\end{array}$ & 23,1 & 0 & 0 \\
\hline $\begin{array}{l}\text { Nivel de autoestima } 6 \\
\text { medio alto }\end{array}$ & 46,2 & 11 & 52,4 \\
\hline $\begin{array}{l}\text { Nivel de autoestima } 4 \\
\text { alto }\end{array}$ & 30,8 & 8 & 38,1 \\
\hline Total & 13 & 100,0 & 21 & 100,0 \\
\hline
\end{tabular}

De acuerdo a los resultados del CETAD "Nuevo Surco" a nivel familiar se determinó que el mayor número de pacientes poseen un nivel de autoestima medio alto con un porcentaje de 46,2 y una frecuencia de 6 , seguido por una frecuencia de 4 equivalente a 30,8 que representa el nivel de autoestima alto, dentro de la tabla se ha anulado el nivel de autoestima bajo debido a que ningún paciente representa ese nivel de autoestima, lo cual evidencia que la relación familiar es percibida como buena misma que ayudará a fomentar otras áreas de los pacientes que presenten un déficit.

Mientras que en el CETAD "CENTRADRO” de acuerdo a los resultados a nivel familiar se determinó que el mayor número de pacientes poseen un nivel de autoestima medio alto con un porcentaje de 52, $4 \%$ y una frecuencia de 11, seguido por una frecuencia de 8 equivalente a $38,1 \%$ que representa el nivel de autoestima alto, dentro de la tabla se ha anulado el nivel de autoestima medio bajo debido a que ningún paciente representa ese nivel de autoestima, lo cual evidencia que la relación familiar es percibida como buena misma que ayudará a fomentar otras áreas de los pacientes que presenten un conflictos que pueden provocar alteraciones en su nivel funcional.

Tabla 6. Distribución de frecuencia y porcentaje de escala general de la escala de autoestima aplicada a los internos de los Centro Especializado en Tratamiento de alcohol y drogas "Nuevo surco" y "CENTRADRO” 2021

\section{Escala General}

\begin{tabular}{l|l|l|l}
\multicolumn{2}{l|}{ Nuevo Surco } & \multicolumn{2}{l}{ CENTRADRO } \\
\hline Frecuenci & Porcentaj & \\
a & e & Frecuencia & Porcentaje \\
\hline
\end{tabular}




\begin{tabular}{ll|l|l|l}
\hline $\begin{array}{l}\text { Válid } \\
\mathrm{0}\end{array}$ & $\begin{array}{l}\text { Nivel de autoestima 1 } \\
\text { bajo }\end{array}$ & 7,7 & 0 & 0 \\
\hline $\begin{array}{l}\text { Nivel de autoestima } 1 \\
\text { medio bajo }\end{array}$ & 7,7 & 1 & 4,8 \\
\hline $\begin{array}{l}\text { Nivel de autoestima } 7 \\
\text { medio alto }\end{array}$ & 53,8 & 12 & 57,1 \\
\hline $\begin{array}{l}\text { Nivel de autoestima } 4 \\
\text { alto }\end{array}$ & 30,8 & 8 & 38,1 \\
\hline Total & 13 & 100,0 & 21 & 100,0 \\
\hline
\end{tabular}

De acuerdo a los resultados del CETAD "Nuevo Surco" a nivel general de autoestima se determinó que el mayor número de pacientes representa el nivel medio alto con una frecuencia de 7 equivalente al 53,8 \% mismo que a pesar de no representar el nivel más alto se convierte en base para fortalecer otras áreas del individuo que presentan dificultad.

Mientras que en el CETAD "CENTRADRO" de acuerdo a los resultados a nivel general de autoestima se determinó que el mayor número de pacientes representa el nivel medio alto con una frecuencia de 12 equivalente al 57,1\% mismo que a pesar de no representar el nivel más alto se convierte en base para fortalecer otras áreas del individuo que presentan dificultad y así también fomentar la misma área hasta alcanzar su nivel máximo logrando que el paciente refuerce esa percepción que posee de sí mismo.

\section{Discusión}

Los resultados anteriormente descritos guardan relación con lo que menciona (Castro, 2012) en su artículo donde describe que el 17.5\% (14) de los pacientes tienen una autoestima inferior, el 50\% (40) una autoestima promedio y el $32.5 \%$ (26) una autoestima superior, equivalentes a los resultados obtenidos dentro de esta investigación donde el autoestima promedio (medio alto) es el que ocupa un mayor porcentaje en ambos centros especializados en el tratamiento de alcohol y drogas.

lo que no guarda relación es que el estudio descrito por la autora mencionada evalúa la autoestima según la etapa del tratamiento donde llega a la conclusión de que la autoestima se incrementa conforme paciente va avanzando en su programa de tratamiento, mientras que en el presente estudio se evalúan las dimensiones de la autoestima ocupando la dimensión de sí mismo el nivel más alto dentro de los pacientes.

La presente investigación describe el nivel de autoestima de dos centros especializados en el tratamiento de alcohol y drogas, mismos que presentan resultados similares en su autoestima, mientras que el estudio 
anteriormente mencionado se enfoca en un solo centro evaluando la autoeficacia, misma que también se incrementa a medida que el paciente avanza en el programa.

\section{Conclusiones}

Descritos los resultados obtenidos del procesamiento de los datos de la escala de autoestima Coopersmith para adultos basándonos en los objetivos se ha llegado a las siguientes conclusiones:

En la escala general de los pacientes internos en los centros especializados en el tratamiento de alcohol y drogas tanto "Nuevo surco" como en "CENTRADRO" obtuvieron un nivel de autoestima medio alto.

Dentro de la dimensión social de los pacientes internos en los centros especializados en el tratamiento de alcohol y drogas tanto "Nuevo surco" como en "CENTRADRO" obtuvieron un nivel de autoestima medio alto.

Mientras que en la dimensión de sí mismo de los pacientes internos en los centros especializados en el tratamiento de alcohol y drogas tanto "Nuevo surco" como en "CENTRADRO" obtuvieron un nivel de autoestima alto.

por ultimo en la dimensión familiar de los pacientes internos en los centros especializados en el tratamiento de alcohol y drogas tanto "Nuevo surco" como en "CENTRADRO” obtuvieron un nivel de autoestima medio alto. Siendo evidente que la autoestima en los centros varía entre nivel alto y medio alto, se lo debe tomar como base para fortalecer áreas de los pacientes que presenten déficit, como consecuencia traerá consigo mejorar la calidad de vida de los mismos.

\section{Referencias bibliográficas}

Apud, I., \& Romani, O. (2016). LA ENCRUCIJADA DE LA ADICCIÓN. DISTINTOS MODELOS EN EL ESTUDIO DE LA DROGODEPENDENCIA. 16, 115-125.

Carbonero, M., Martin, L., \& Feijo, M. (2010). Las creencias irracionales, en relación con ciertas conductas de consumo en adolescentes. https://doi.org/10.30552/ejep.v3i2.57

Castro, L. (2012). AUTOESTIMA Y AUTOEFICACIA EN PACIENTES ADICTOS EN EL CENTRO DE REHABILITACION DE ÑANA. http://www.hhv.gob.pe/wp-content/uploads/Revista/2012/2012_12_01.pdf

COOPERSMITH. (2010). Academia. Recuperado el 15 de Noviembre de 2017, de Academia: http://www.academia.edu/3093021/TEST_DE_COOPERSMITH_ADULTOS

Cuero, M. (2017). Niveles de autoestima en los estudiantes de décimo que inician el consumo de alcohol [UNIVERSIDAD CENTRAL DEL ECUADOR]. http://www.dspace.uce.edu.ec/bitstream/25000/13357/1/TUCE-0007-PC023-2017.pdf 
Gámez, M., \& Guzmán, F. (2017). Autoestima y consumo de alcohol en adolescentes escolarizados. https://www.nureinvestigacion.es//OJS/index.php/nure/article/view/885

Liberini, S., Rodriguez, G., \& Romero, N. (2016). EL ROL DE LA AUTOESTIMA, LA PERSONALIDAD Y LA FAMILIA EN EL CONSUMO DE ALCOHOL EN LA ADOLESCENCIA. 27-37.

Loor, W., \& Hidalgo, H. (2018). Causas de las adicciones en adolescentes y jóvenes en Ecuador. 22, 130-138.

Matrangolo, G., Yaccarini, C., \& Paz, G. (2017). AUTOESTIMA, PERSONALIDAD, ESPIRITUALIDAD Y CENTRALIDAD DE LOS EVENTOS TRAUMÁTICOS VINCULADOS A LAS PROBLEMÁTICAS PSICOSOCIALES EN LA INFANCIA. https://datahub.io/dataset/2017-2-2-e33

Misitu Ochoa, G., \& Herrero Oilazola, J. (2003). El rol de la autoestima en el consumo moderado de drogas en la adolescencia. Revista Internacional de Ciencias Sociales y Humanisticas SOCIOTAM, 285-306.

Moreno, E. (2016). Plan terapéutico para adicciones basadas en terapia cognitivo conductual. [Universidad Técnica de Machala.]. http://186.3.32.121/bitstream/48000/8629/1/ECUACS\%20DE00029.pdf

Naranjo Pereira, M. (2007). Autoestima: un factor relevante en la vida de la persona y tema esencial del proceso educativo. Actualidades Investigativas en Educacion, https://www.redalyc.org/articulo.oa?id=447/44770311. Telumbre, J., López, M., \& Noh, P. (2018). Autoestima y consumo de alcohol en adolescentes de secundaria de Ciudad del Carmen, Campeche. 20-31.

Tena, A., Castro, G., Marín, R., \& Gómez, P. (2018). Consumo de sustancias en adolescentes: Consideraciones para la práctica médica. http://www.scielo.org.mx/scielo.php?pid=S0186$48662018000200008 \&$ script=sci_arttext

Villarreal, M., \& Sanchez, J. (2010). El Consumo de Alcohol en Adolescentes Escolarizados: Propuesta de un Modelo Sociocomunitario. 19. http://scielo.isciii.es/scielo.php?script=sci_arttext\&pid=S113205592010000300006 